\title{
BvDU Kurz notiert
}

\section{Berufsverband der Deutschen Urologen e.V.}

Urologe $2020 \cdot 59: 878$

https://doi.org/10.1007/s00120-020-01257-y

(c) Springer Medizin Verlag GmbH, ein Teil von Springer Nature 2020
Redaktion

Dr. R. Zielke (V.i.S.d.P.)

BvDU-Geschäftsführer

Kantstr. 149

10623 Berlin

\section{Gleiches Geld für gleiche Leistung?}

\begin{abstract}
Medizinische Leistungen wie z.B. Operationen, die sowohl ambulant als auch stationär durchgeführt werden können, werden bislang je nach Sektor unterschiedlich bezahlt. Dadurch besteht die Möglichkeit, dass der Patient nicht im für ihn angemessenen Sektor behandelt wird. Wie muss ein einheitliches, übergreifendes Vergütungssystem ausgestaltet sein, damit die in verschiedenen Sektoren gleich erbrachten Leistungen berücksichtigt und Fehlsteuerungen vermieden werden können? Das untersucht jetzt ein vom Innovationsfonds des Gemeinsamen Bundesausschusses mit 1,1 Millionen Euro gefördertes Projekt unter Leitung des Hamburg Center for Health Economics (HCHE) der Universität Hamburg
\end{abstract}

\section{Quelle: Hamburg Center for Health Economics (HCHE)}

\section{Störung in der Telematikinfrastruktur}

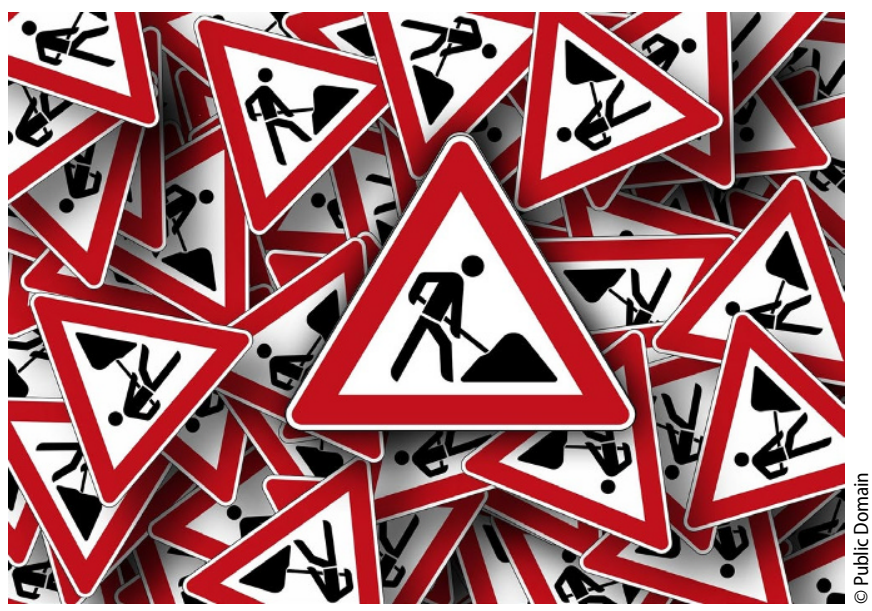

Ende Mai gab es in der Telematikinfrastruktur (TI) eine größere Störung: In vielen Praxen war damit kein Online-Abgleich von Versichertenstammdaten (VSDM) möglich. Nach ersten Schätzungen sind rund 80.000 Konnektoren in den Praxen von Ärzten, Psychotherapeuten und Zahnärzten betroffen.

Zu dem Ausfall der Konnektoren hat nach Mitteilung der gematik ein Konfigurationsfehler in der zentralen TI geführt. Um das Problem zu beheben, müssen jetzt alle Konnektoren manuell aktualisiert und damit für den Online-Betrieb wieder freigeschaltet werden. Das genaue Vorgehen ist abhängig vom jeweiligen Konnektor-Hersteller. Die Praxen sollten sich deshalb mit ihrem Dienstleister in Verbindung setzen. Informationen dazu stellt die gematik auf ihrer Internetseite bereit, die sie fortlaufend aktualisieren will. Zum Redaktionsschluss war das Ausmaß der Folgen nicht in Gänze bekannt. Die KBV ist im Gespräch mit der gematik und dem Bundesgesundheitsministerium, um den Praxen schnell und unbürokratisch zu helfen. Die KBV fordert, dass den Ärzten und Psychotherapeuten dadurch keinerlei Nachteile entstehe. Sie dürften weder mit Regressen überzogen werden, weil sie für die Krankenkassen das VSDM nicht durchführen können, noch dürften sie auf irgendwelchen Kosten sitzen bleiben. Da es sich um einen zentralen Konfigurationsfehler handele, würden die Praxen für den Remote- beziehungsweise Vor-Ort-Termin nicht zahlen. Dafür will sich die KV vehement einsetzen.

Quelle: KBV 